\title{
PROGRAMA PARA MEJORAR LAS HABILIDADES SOCIO- COGNITIVAS CON ALUMNOS DE DIFERENTES CULTURAS EN LA CIUDAD AUTÓNOMA DE MELILLA ${ }^{1}$
}

\author{
Dolores Seijo Martínez ${ }^{2}$ \\ Mercedes Novo Pérez ${ }^{3}$ \\ Laila Mohamed Mohand ${ }^{4}$
}

\begin{abstract}
Social and cognitive skills are founded on perspectives of positive development in young people. In this regard, the resources that they must have at their disposal in order to become competent adults have been central to research in this area (Gutierrez; Escartí, Pascual, 2011). We must consider socio-cognitive skills within a cultural and contextual framework, taking into account the communication patterns of each culture, given the great variety of these across and within cultures. This paper highlights the importance that socio-cognitive skills may have in achieving harmonious relations among schoolchildren, which will favor the teaching-learning process. To this end, we present a summary of the design and implementation of a program whose aim was to prevent disruptive behavior in schools. The objectives of our intervention were: social and communication skills, development of values, problem-solving, creative thinking, and emotional control, all of which are linked to the development of pro-social behavior (Seijo; Novo; Arce; Fariña; Mesa, 2005). Intervention was conducted with a sample of 441 Elementary and Secondary students, from four schools in the Autonomous City of Melilla. Finally we discuss the main results of our study.
\end{abstract}

Keywords: training in social skills; intercultural population; education; disruptive behavior

Resumen: Las habilidades sociales y cognitivas se encuentran en la base de la perspectiva del desarrollo positivo de los jóvenes, y en este sentido los recursos que deben ser aprendidos para convertirse en adultos competentes han sido tema central de estudio para poder alcanzar este desarrollo positivo (Gutiérrez; Escartí; Pascual, 2011). Asimismo, las habilidades y destrezas socio-cognitivas deben ser consideradas atendiendo al marco cultural y contextual, teniendo en cuenta los patrones de comunicación propios de cada cultura, dada la alta variabilidad de éstos entre culturas e incluso dentro de una misma cultura en diferentes contextos. El trabajo que se presenta trata de poner de relieve la importancia que las habilidades socio-cognitivas pueden tener en el alcance de una convivencia adecuada entre los escolares, que favorezca el proceso de enseñanza-aprendizaje. De este modo, se presenta un resumen del diseño e implementación de un programa de prevención de

Seijo Martínez, D.; Novo Pérez, M.; Mohamed Mohand, L. (2012). Programa para mejorar las habilidades socio-cognitivas con alumnos de diferentes culturas en la Ciudad Autónoma de Melilla. DEDiCA. REVISTA DE EDUCAÇÃO E HUMANIDADES, 3 (2012) março, 229-242 
comportamientos disruptivos en el ámbito escolar. Los contenidos objeto de intervención en el programa son: habilidades sociales y de comunicación, desarrollo de valores, solución de problemas, pensamiento creativo y control emocional, todos vinculados con el desarrollo de comportamientos prosociales (Seijo; Novo; Arce; Fariña; Mesa, 2005). La intervención se llevó a cabo con una muestra total de 441 alumnos del último ciclo de Educación Primaria y primer ciclo de Educación Secundaria, pertenecientes a cuatro centros educativos de la Ciudad Autónoma de Melilla. Finalmente se discuten los principales resultados de este estudio.

Palabras claves: entrenamiento en habilidades sociales; población intercultural; educación; comportamiento disruptivo

\section{Introducción}

Recientes estudios (Bravo; Herrera, 2011, García, 2011) muestran la necesidad de detectar lo más temprano que sea posible los problemas de convivencia en el aula así como los déficits de los alumnos, para poder intervenir de manera precoz y facilitar el trabajo de profesores y educadores en el proceso de enseñanzaaprendizaje.

Hasta la actualidad se han dado numerosas definiciones sobre las habilidades sociales sin llegar a un acuerdo sobre lo que es una conducta socialmente habilidosa. La definición aportada por Caballo (2002, p.6) es la que más peso está teniendo en este campo, ya que como el autor señala: "el comportamiento socialmente habilidoso es un conjunto de conductas realizadas por un individuo en un contexto interpersonal que expresa sentimientos, actitudes, deseos, opiniones o derechos de un modo adecuado a la situación, respetando esas conductas en los demás, y que generalmente resuelve los problemas inmediatos de la situación mientras reduce la probabilidad de que aparezcan futuros problemas".

Las habilidades sociales deben ser consideradas dentro de un marco cultural determinado, y los patrones de comunicación varían entre culturas y dentro de una misma cultura, dependiendo de factores como la edad, el género, la clase social y la educación (Caballo, 2002). Este autor también afirma que saber cuándo y cómo se aprenden las habilidades sociales es una incógnita, pero que la niñez es sin duda un período crítico. Así podemos asegurar que los niños más inhibidos tienen menos oportunidades de aprender y practicar conductas sociales y, por consiguiente, adquieren menos refuerzos bajo la forma de alabanzas, sonrisas 0 caricias por parte de las personas de su entorno. 
La importancia de la adquisición de las habilidades sociales por parte de un individuo, radica en la necesidad de mantener con los demás una relación interpersonal en la que se manifieste de manera cómoda y adecuada.

El ser humano es social por naturaleza y necesita tener una estrecha relación con el ambiente social en el que se encuentra. El ser conscientes en la vida de que el comportamiento que manifiesta uno mismo y el que muestra para con los demás puede ser cambiado, así como conocer los medios para que esto se produzca, hace que se de más importancia a la adquisición de las habilidades sociales y sobre todo a su entrenamiento.

Si una persona no sabe comportarse de forma habilidosa con los demás, debe aprender a hacerlo para que su relación sea exitosa, pero muchas veces ese intento se ve frustrado debido a que, en algunas situaciones, el sujeto se siente bloqueado, ansioso o tiene una necesidad imperiosa de evitar esa situación incómoda para él, en este caso estos problemas rompen la relación que se iba a establecer y como consecuencia no existe ningún tipo de relación interpersonal y no se efectúa dicho comportamiento. Lo que lleva a resaltar, que la existencia o aparición de una serie de trastornos psicológicos que pueda sufrir el ser humano a lo largo de su vida (depresión, ansiedad,...) hace que sea imprescindible el entrenamiento en habilidades sociales para afrontar estos problemas.

Antes de poner en práctica nuestras habilidades sociales, debemos analizar y estudiar el medio en el que nos hallamos, para así evitar situaciones comprometidas y salir airoso de ese encuentro. Cuando queremos saber cuales son los problemas que debemos afrontar al tener una relación interpersonal, debemos tener en cuenta cuál es el contexto en el que nos encontramos y en el que se va a llevar a cabo esa relación. Los contextos en los que el niño se desarrolla, por definición son: el familiar, el escolar y el social.

La familia es el grupo social donde surgen las primeras manifestaciones de conductas sociales y afectivas, por lo que la manera en la que unos padres se relacionan con sus hijos, es de vital importancia para saber hasta qué punto, esta relación podrá favorecer el desarrollo de las competencias sociales en sus hijos y les ayuden a ser más responsables y poseer una autoestima elevada y que así pongan en práctica estrategias de solución de problemas. La importancia de todo esto radica, en que es dentro del grupo familiar, donde se lleva a cabo la primera adaptación del niño 
a la vida social, si ésta resulta de gran satisfacción para él, en el futuro tendrá muchas posibilidades de que su adaptación y socialización en la vida sea exitosa. Cuando el niño comienza a mantener algún tipo de relación interpersonal con los demás, éste presenta una serie de conductas de proximidad y contacto que determinan el principio de sus habilidades sociales. Todo esto supone el inicio del proceso de socialización que, como hemos indicado anteriormente, se lleva en primer lugar por la familia, quien inicia el proceso para la formación de habilidades sociales. Tanto el niño como el adolescente deben utilizar sus habilidades por muy complejas que éstas sean para poder ser un individuo social y así aprender de sus propios errores en el caso de no utilizar las adecuadas en cada situación.

Seguidamente, con la incorporación del niño al sistema educativo, éste desarrolla habilidades más complejas y específicas a lo largo de las distintas etapas de su vida lo que le va a proporcionar, ciertas exigencias, donde ocupa un lugar importante las relaciones que se establecen con el grupo de iguales, y es aquí donde debe ponerlas en práctica al interaccionar con ellos (compañeros), además de tener que aprender normas sociales y saber distinguir entre un comportamiento adecuado e inadecuado. El que el niño se sienta perteneciente a un grupo, facilita el desarrollo de su propia identidad. Por lo que el niño en la escuela, en definitiva, desarrolla habilidades más complejas para poder ser un sujeto social.

En los últimos años estamos notando en la sociedad un incremento en la manifestación de comportamientos antisociales y disruptivos. La aparición y desarrollo de dichos comportamientos es una variable que debe ser valorada a la hora de plantear un programa de cara a la prevención, puesto que como indican números autores (Farrington; West, 1990; Hoeve; Blokland; Dubas; Loeber;Gerris; Van der Lann, 2008; Redondo; Pueyo, 2007), pueden ser predictores de futuras conductas delictivas. Múltiples investigaciones señalan las variables externas al individuo tales como el contexto social, contexto familiar, exposición a la violencia y oportunidades para delinquir como factores que provocan la aparición del comportamiento antisocial (Andreu; Peña; Larroy, 2010; Timmerman; Emmelkamp, 2005).

De este modo, se debe concienciar a los menores sobre que el manejo de habilidades y estrategias socialmente competentes permite relacionarse de una manera adecuada con las personas que 
le rodean, disminuyendo así la manifestación de comportamientos disruptivos. El alumno encuentra en las habilidades sociales las herramientas necesarias para enfrentarse y poder solucionar los problemas que se le puedan presentar (Goldstein; Sprafkin; Gershaw; Klein, 1989).

Recientes estudios (Bravo; Herrera, 2011, García, 2011) constatan la necesidad de detectar lo más temprano que sea posible los problemas de convivencia en el aula así como los déficits de los alumnos, para poder intervenir de manera precoz y facilitar el trabajo de profesores y educadores en el proceso de enseñanzaaprendizaje. Con este trabajo se ha pretendido hacer frente a los posibles problemas que puedan tener los alumnos de Educación Primaria y primera etapa Educación Secundaria Obligatoria al no saber utilizar correctamente sus habilidades sociales. Para ello, se pondrá en práctica un programa de entrenamiento en habilidades sociales, en estas etapas educativas. El programa tiene como principal objetivo la prevención de comportamientos disruptivos en contextos escolares. Defiende que mejorar las habilidades sociocognitivas es una forma de ayudar a los menores a evitar la desadaptación personal y la desviación social. Se basa en el entrenamiento de diferentes contenidos relacionados con la promoción de la competencia social positiva (Ross et al. 1990). Estos contenidos abordan dos dimensiones, social y cognitiva.

En la primera entrenamos habilidades sociales y de comunicación, mientras que, enmarcadas en la vertiente cognitiva, se desarrolla pensamiento creativo, control emocional, valores y solución de problemas. Este programa asume que la organización escolar es un ámbito adecuado para intervenir con los adolescentes, especialmente para la prevención de conductas sociales disruptivas porque permite trabajar con toda la población, posibilita la integración de los profesionales de los centros (maestros, profesores, pedagogos, psicopedagogos y psicólogos) en el programa y permite una apertura hacia la intervención en los ámbitos familiar y social. La propuesta es una adaptación del modelo de entrenamiento cognitivo-conductual de Ross y otros (1990) que se centra en las habilidades cognitivas y actitudes que la investigación ha establecido como fundamentales para la competencia social (Ross; Ross, 1995).

En la dimensión social, tal y como hemos adelantado, se han trabajado habilidades sociales, puesto que son herramientas necesarias para poder desenvolverse en la vida diaria, por tanto, su 
enseñanza y aprendizaje se considera primordial y necesaria en edades tempranas y su déficit se relaciona directamente con una baja aceptación y aislamiento social, problemas dentro del ámbito educativo y desajustes personales. Asimismo, se han abordado, en varias sesiones, habilidades de comunicación, dado que se consideran fundamentales para el desarrollo y funcionamiento adaptativo del individuo, por lo que un déficit en este tipo de estrategias contribuye a provocar disfunciones y perturbaciones psicológicas.

Dentro de la dimensión cognitiva se han trabajado varios bloques de contenidos que iremos comentando. De este modo, se ha llevado un entrenamiento en pensamiento creativo, cuyo objetivo pretende romper la rigidez cognitiva de los alumnos enseñándoles un pensamiento que les ayude a ser y pensar eficazmente. En el bloque de solución de problemas, se facilita al alumno una visión general del problema, la exposición de las diferentes alternativas, la toma de decisiones y la implementación de la solución elegida, y en el caso de no obtener los resultados esperados, disponer de las estrategias necesarias para emprender un nuevo procedimiento que mejore al anterior. De suma importancia también es el bloque de desarrollo de valores, puesto que los valores reflejan la forma de ser de un individuo, representan la creencia y convicción razonada y firme de que algo es bueno o malo y lo que conviene que hagamos, es decir los valores hacen que nuestra conducta sea adaptada, nos ayudan a entendernos y entender a los demás. Finalmente, trabajamos control emocional con el objetivo de que los sujetos sean conscientes de que en muchas ocasiones nuestra forma de ser, el ponernos nerviosos o enfadarnos funciona en detrimento de la eficaz solución de los problemas.

La Dirección Provincial del Ministerio de Educación, Cultura y Deporte y la Consejería de Educación de la Ciudad Autónoma de Melilla han posibilitado que este programa se lleve a la práctica, de forma piloto, en varios centros escolares de esta ciudad.

A continuación señalaremos las actividades concretas que se han desarrollado durante la intervención. Un profundo desarrollo y explicación de las mismas podemos encontrarlo en Seijo; Novo; Fariña; Arce; Mesa (2004).

\section{Técnicas de intervención}

Para llevar a cabo el correcto desarrollo del programa se considera necesario un buen dominio de las siguientes técnicas: 
instrucciones, modelado, role-playing, retroalimentación, refuerzo y entrenamiento en generalización (ver tabla 1).

\begin{tabular}{|l|l|}
\hline \multicolumn{1}{|c|}{ Técnicas } & \multicolumn{1}{c|}{ Objetivos } \\
\hline Instrucciones & Informar al sujeto de cómo debe realizarse la conducta. \\
\hline Modelado & Observar cómo debe realizarse una conducta. \\
\hline $\begin{array}{l}\text { Role-playing o } \\
\text { dramatización }\end{array}$ & Practicar la conducta para aprenderla. \\
\hline Retroalimentación & Informar sobre la ejecución. \\
\hline Reforzamiento & Conseguir el fortalecimiento de la respuesta. \\
\hline $\begin{array}{l}\text { Reestructuración } \\
\text { cognitiva }\end{array}$ & $\begin{array}{l}\text { Cambiar pensamientos irracionales por otros más } \\
\text { adaptados }\end{array}$ \\
\hline Tareas para casa & Practicar las conductas entrenadas en contextos naturales \\
\hline
\end{tabular}

Tabla 1. Técnicas utilizadas en el programa (Adaptada de Vallés y Vallés, 1996)

\section{Actividades del programa de intervención}

A través de este programa se entrenan aquellas variables relacionadas con el comportamiento prosocial (Mesa; Mohamed; Mohamed; Vázquez, 2003) siendo el entrenamiento en habilidades sociales uno de los pilares fundamentales del mismo (Seijo; Mesa; Mohamed; Mohamed, 2004).

Las actividades que se diseñaron en este programa se adaptaron a la etapa educativa de los alumnos (Primaria 0 Secundaria) teniendo en cuenta, además, el contexto intercultural de la Ciudad Autónoma de Melilla. Las sesiones de actividades están dirigidas por dos técnicos que actúan siempre en pareja, con formación en psicopedagogía y con entrenamiento específico en las técnicas que se han de utilizar en el programa que anteriormente han sido mencionadas. Además, siempre que sea oportuno se tratará de encomendar tareas para casa con la finalidad de practicar las conductas entrenadas en contextos naturales.

A continuación, se recogen las actividades que se han desarrollado en el programa. En total se realizaron 30 actividades en Educación Primaria y 28 en Educación Secundaria.

Los contenidos abordados en Educación Primaria y Educación Secundaria han sido en la mayoría de las ocasiones los mismos, modificando bien el grado de dificultad requerido o bien la adaptación a la edad dependiendo de la etapa educativa a las que se dirigen.

Tanto en Educación Primaria como Educación Secundaria se trabajaron los siguientes contenidos (Tabla 2), mediante las actividades que se especifican seguidamente (para mayor información véase Seijo et al., 2005 a y b): 


\begin{tabular}{|c|c|c|}
\hline CONTENIDOS & EDUCACIÓN PRIMARIA & EDUCACIÓN SECUNDARIA \\
\hline $\begin{array}{l}\text { HABILIDADES } \\
\text { SOCIALES }\end{array}$ & $\begin{array}{l}\text {-Yo soy así. } \\
\text { - Conocer mi autoestima. } \\
\text { - Trabajar mi autoestima. } \\
\text {-Conducta pasiva, asertiva } \\
\text { y agresiva. } \\
\text { - Así soy yo. } \\
\text {-Conociendo a mis } \\
\text { compañeros. } \\
\text { - El careo. } \\
\text { - La caja tonta. } \\
\text { - Personajes de clase. } \\
\text {-Canción } \\
\text { esperanza". color }\end{array}$ & $\begin{array}{l}\text { - ¿Cuál es tu conducta? } \\
\text {-Conducta pasiva, asertiva y } \\
\text { agresiva. } \\
\text { - Actividad grupal. } \\
\text { - Tarea para casa. } \\
\text { - Así soy yo. } \\
\text {-Conociendo a mis } \\
\quad \text { compañeros. } \\
\text { - El careo. } \\
\text { - La caja tonta. } \\
\text { - Personajes de clase. }\end{array}$ \\
\hline $\begin{array}{l}\text { HABILIDADES } \\
\text { DE } \\
\text { COMUNICACIÓN }\end{array}$ & $\begin{array}{l}\text { - Técnica del rumor. } \\
\text { - Rostros y posturas. }\end{array}$ & $\begin{array}{l}\text { - Técnica del rumor. } \\
\text { - Rostros y posturas. }\end{array}$ \\
\hline $\begin{array}{l}\text { PENSAMIENTO } \\
\text { CREATIVO }\end{array}$ & $\begin{array}{l}\text { - Aspectos positivos, } \\
\text { negativos } \\
\text { interesantes. } \\
\text { - Adivinanzas. } \\
\text { - Fotopalabra. } \\
\text { - Buzón de sugerencias. } \\
\text { - Análisis crítico. } \\
\text { - Canciones. } \\
\text { - Programa de radio. }\end{array}$ & $\begin{array}{l}\text { - Aspectos positivos, } \\
\text { negativos e interesantes. } \\
\text { - Acertijos. } \\
\text { - Fotopalabra. } \\
\text { - Buzón de sugerencias. } \\
\text { - Análisis crítico. } \\
\text { - Canciones. } \\
\text { - Programa de radio. }\end{array}$ \\
\hline $\begin{array}{l}\text { SOLUCIÓN } \\
\text { DE } \\
\text { PROBLEMAS }\end{array}$ & $\begin{array}{l}\text { - Lluvia de ideas. } \\
\text {-¿Cuáles son tus } \\
\text { problemas? } \\
\text {-¿Cuáles son las } \\
\text { consecuencias? } \\
\text { - Acciones } \\
\text { consecuencias a corto y y } \\
\text { largo plazo. } \\
\text {-Solución de problemas } \\
\text { específicos. }\end{array}$ & $\begin{array}{l}\text { - Lluvia de ideas. } \\
\text { - ¿Cuáles las } \\
\text { consecuencias? } \\
\text { - Acciones y consecuencias a } \\
\text { corto y largo plazo. } \\
\text {-Solución de problemas } \\
\text { específicos. }\end{array}$ \\
\hline $\begin{array}{l}\text { DESARROLLO } \\
\text { DE } \\
\text { VALORES }\end{array}$ & $\begin{array}{l}\text { - Ranking de valores. } \\
\text { - Taller de riqueza } \\
\text { intercultural. } \\
\text { - Dilemas morales. } \\
\text { - Juego de rol. } \\
\text { - Valor oculto. }\end{array}$ & $\begin{array}{l}\text { - Ranking de valores. } \\
\text { - Taller de riqueza } \\
\text { intercultural. } \\
\text { - Dilemas morales. } \\
\text { - Juego de rol. }\end{array}$ \\
\hline $\begin{array}{l}\text { CONTROL } \\
\text { EMOCIONAL }\end{array}$ & - Situaciones de conflicto. & - Situaciones de conflicto. \\
\hline
\end{tabular}

Tabla 2. Actividades desarrolladas en el programa de entrenamiento en habilidades socio-cognitivas (Seijo, Novo, Fariña, Arce y Mesa, 2004)

\section{Diseño}

Se han elegido al azar 16 aulas de cuatro centros escolares de la Ciudad Autónoma de Melilla, para implementar este programa experimental. Dos de ellos de Educación Primaria (centro 1 y centro 
2) y dos de Educación Secundaria (centro 3 y centro 4). Los centros escolares fueron elegidos, de tal manera que por su ubicación geográfica representarán a la población melillense en general. En total, han participado una muestra total de 441 alumnos, de los cuales 200 pertenecen a Educación Primaria y 241 a Educación Secundaria (tabla 3).

\section{Metodología}

La metodología de investigación empleada fue del tipo cuasi-experimental y en un ambiente natural. La investigación se ajusta a un diseño pre-post con grupo control de comparación (Pereda, 1987). El instrumento de medida utilizado es la Escala de Razonamiento Moral.

La muestra en cuanto a la condición experimental se distribuye en 235 alumnos del grupo experimental y 206 del grupo control (ver tabla 3). Se ha ejecutado una prueba t de Student para muestras relacionadas tanto para las variables de tipo social como cognitivas.

\begin{tabular}{|l|l|c|c|c|}
\cline { 3 - 5 } \multicolumn{2}{c|}{} & EXPERIMENTAL & CONTROL & TOTAL \\
\hline \multirow{2}{*}{$\begin{array}{l}\text { Educación } \\
\text { primaria }\end{array}$} & Centro 1 & 52 & 57 & 109 \\
\cline { 2 - 5 } & Centro 2 & 60 & 31 & 91 \\
\hline \multirow{2}{*}{$\begin{array}{l}\text { Educación } \\
\text { secundaria }\end{array}$} & Centro 3 & 69 & 68 & 137 \\
\cline { 2 - 5 } & Centro 4 & 54 & 50 & 104 \\
\hline & TOTAL & 235 & 206 & 441 \\
\hline
\end{tabular}

Tabla 3. Distribución de la muestra por centros, nivel educativo y condición experimental

\section{Resultados y conclusiones}

Teniendo como referencia la vertiente social y cognitiva que hemos descrito, señalaremos los resultados más relevantes de esta investigación (Seijo et al, 2005 a y b). En primer lugar, no estamos en condiciones de establecer conclusiones sobre la eficacia del programa en la parte más social (Mohamed-Mustafa, 2004). Esto es, no podemos conocer si las actividades sobre habilidades sociales y de comunicación que hemos desarrollado con los alumnos han funcionado 0 no. Esto ha sido debido fundamentalmente a cuestiones como la selección del instrumento de medida o la falta de sinceridad de los alumnos. Otra variable que, con toda seguridad ha afectado, ha sido la valoración por parte de los tutores, dado que tenían que evaluar a sus alumnos antes de la intervención y una vez finalizada la misma, y los profesores no eran los mismos en ambos 
momentos, lo que ha implicado una falta de fiabilidad interjueces y por tanto un desajuste en los resultados. De hecho, presumimos que las actividades que se han desarrollado han sido válidas dado que van en la línea de las propuestas en otros programas (Garrido; López, 1995; Rodríguez; Luca de Tena, 2001; Trianes; FernándezFiguares, 2001; Vallés; Vallés, 1997; 1994) que han demostrado su eficacia. En definitiva, habría que poner a prueba de nuevo estas actividades y comprobar su adecuación utilizando otros instrumentos de medida.

Por otra parte, para el diseño de las actividades respecto a la dimensión cognitiva nos hemos basado en otras intervenciones (Díaz-Aguado, 1999, Garrido; López, 1995) adaptándolas de forma rigurosa al contexto social de Melilla. Podemos adscribir a esta dimensión las actividades de desarrollo de valores, pensamiento creativo, control emocional y solución de problemas. De esta manera, se ha conseguido que los alumnos participantes en la intervención avancen en el estadio de desarrollo moral (tablas 4 y 5), lo cual implica necesariamente una mejora en el desarrollo cognitivo constatando la eficacia de la intervención (Mohamed-Mohand, 2004). De igual modo, cada estadio de desarrollo moral se asocia a un determinado modo de pensar sobre temas socio-morales y un avance se relaciona con un cambio cualitativo en el razonamiento. Este cambio va, necesariamente, acompañado con una reestructuración de pensamiento. Los estadios forman secuencias invariantes determinados por la complejidad lógica de cada etapa. Esto es, para que un estadio más avanzado se alcance es necesario dominar operaciones cognitivas previas que permitan desarrollar otras más complejas. En definitiva, estamos en condiciones de afirmar que los alumnos han avanzado en desarrollo moral, lo que implica necesariamente un avance en el desarrollo cognitivo. Estos resultados van en la dirección de los encontrados por Bravo y Herrera (2011) quienes destacan que la enseñanza de habilidades sociales y cognitivas contribuye a la formación integral de los jóvenes y a su desarrollo personal y social.

\begin{tabular}{|c|c|c|c|c|}
\hline Variable & $\mathrm{t}$ & $p$ & $M_{P R E}$ & MPOST \\
\hline $\begin{array}{l}\text { Razon. Moral (grupo } \\
\text { experimental) }\end{array}$ & -4.124 & .000 & 1.87 & 2.32 \\
\hline $\begin{array}{l}\text { Razon. Moral (grupo } \\
\text { control) }\end{array}$ & -1.000 & .322 & 1.40 & 1.50 \\
\hline
\end{tabular}

Tabla 4. Comparación pre-post en la Escala de Razonamiento Moral en Educación Primaria 


\begin{tabular}{|l|c|c|c|c|}
\hline \multicolumn{1}{|c|}{ Variable } & $\mathrm{t}$ & $\mathrm{p}$ & $\mathrm{M}_{\text {PRE }}$ & $\mathrm{M}_{\text {POST }}$ \\
\hline $\begin{array}{l}\text { Razon. Moral (grupo } \\
\text { experimental) }\end{array}$ & -5.112 & .000 & 2.04 & 2.60 \\
\hline $\begin{array}{l}\text { Razon. Moral (grupo } \\
\text { control) }\end{array}$ & 1.386 & .173 & 2.13 & 1.95 \\
\hline
\end{tabular}

Tabla 5. Comparación pre-post en la Escala de Razonamiento Moral en Educación Secundaria

Para finalizar, defendemos la pertinencia de los programas de entrenamiento multimodales (esto es, cognitivo-conductuales) en competencia social (Arce; Seijo; Fariña; Mohamed-Mohand, 2010, Mohamed-Mohand, 2008) y teniendo en cuenta numerosas variables (i.e.: la educación parental, el aprendizaje de habilidades sociales, el manejo de la ira o enfado, solución de conflictos, educación en la escuela sobre la violencia, iniciativas de autoridad, liderazgo y mediación con los iguales, organización de la comunidad y campañas de los medios de comunicación, etc.) que pueden estar incidiendo en la prevención primaria de los comportamientos antisociales en los jóvenes. Por tanto sería idóneo poder actuar sobre todas ellas, pero evidentemente, la puesta en marcha de una intervención susceptible de manejar todas estas variables conllevaría una fuerte implicación institucional, acompañada de una enorme dotación económica, lo cual sería altamente deseable. Sin embargo, somos conscientes de la dificultad, cuando no imposibilidad, de alcanzar grandes presupuestos para el desarrollo de programas de esta naturaleza. De este modo, dadas las limitaciones, nuestra propuesta sigue asumiendo que la organización escolar es un ámbito adecuado para intervenir con los adolescentes, sin olvidar la importancia de una intervención integral, esto es, que abarque no sólo los ámbitos personal, social y escolar sino también el familiar. En este sentido sugerimos que para que el programa sea eficaz en su totalidad sería imprescindible proceder a una labor de formación e implicación efectiva del profesorado de tal forma que ellos mismos estuviesen en condiciones de desarrollar durante cada curso escolar las actividades previstas en el programa. Sin olvidar también la importante labor de los profesores en la implicación de los padres y otros responsables de los menores en las actividades diseñadas en el marco del programa.

\section{Referencias Bibliográficas:}

Andreu, J. M.; Peña, M. E.; Larroy, C. (2010). Conducta antisocial, impulsividad y creencias justificativas: análisis de sus interrelaciones con la 
agresión proactiva y reactiva en adolescentes. Behavioral Psychology, 18 (2010) 57-72.

Arce, R; Seijo, D.; Fariña, F.; Mohamed-Mohand, L. (2010). Comportamiento antisocial en menores: riesgo social y trayectoria natural de desarrollo. Revista Mexicana de Psicología, 27, 2 (2010) 127-142.

Bravo, I.; Herrera, L. (2011). Convivencia escolar en Educación primaria. Las habilidades Sociales del alumnado como variable moduladora. DEDiCA. REVISTA DE EDUCAÇÃO E HUMANIDADES, 1 (2011) Março, 173-212.

Caballo, V. E. (2002). Manual de evaluación y entrenamiento de habilidades sociales. Madrid: Siglo XXI.

Díaz-Aguado, M. J. (1999). Programas de educación para la tolerancia y prevención de la violencia en los jóvenes. Madrid: Ministerio de Trabajo y Asuntos Sociales. Instituto de la Juventud.

Elzo, J. (1999). Jóvenes españoles 1999. Madrid: Narcea.

Farrington, D. P.; West, D. J. (1990). The Cambridge study in delinquent development: A long-term follow-up of 411 London males. In H. J. Kerner; G. Kaiser (Eds.), Criminality: Personality, Behaviour and Life History, 115-38. Heidelberg: Springer-Verlag.

García, P. (2011). Análisis de la convivencia escolar en aulas de educación primaria. Revista Iberoamericana de Educación, 55, 3 (2011) 112.

Garrido, V.; López, M. J. (1995). La prevención de la delincuencia: el enfoque de la competencia social. Valencia: Tirant lo Blanch.

Goldstein, A. P.; Sprafkin, R. P.; Gershaw, N. J.; Klein, P. (1989). Habilidades sociales y autocontrol en la adolescencia. Un programa de enseñanza. Barcelona: Martínez Roca.

Gutierrez, M.; Escartí, A.; Pascual, C. (2011). Relaciones entre empatía, conducta prosocial, agresividad, autoeficacia y responsabilidad personal y social de los escolares. Psicothema, 23, 1 (2011) 13-19.

Hoeve, M.; Blokland, A.; Dubas, J. S.; Loeber, R.; Gerris, J. R. M.; Van der Laan, P. H. (2008). Trajectories of delinquency and parenting styles. Journal of Abnormal Child Psychology, 36, 2 (2008) 223-235.

Mesa, M. C.; Mohamed-Mustafa, N.; Mohamed-Mohand, L.; Vázquez, M. J. (2003). Variables predictoras del comportamiento violento en jóvenes escolares: factores de riesgo. Comunicación presentada a las VIII Jornadas Andaluzas de Organización y Dirección de Instituciones Educativas. Celebrado en Granada del 15 al 17 de diciembre.

Mohamed-Mohand, L. (2004). Intervención socio-cognitiva con menores en educación secundaria: entrenamiento en desarrollo de valores. Trabajo de investigación tutelada no publicado: Universidad de Granada.

Mohamed-Mohand, L. (2008). Estudio de campo del comportamiento inadaptado en menores: riesgo social y evolución natural. Tesis doctoral no publicada: Universidad de Granada. 
Mohamed-Mustafa, N. (2004). Intervención socio-cognitiva con menores en educación primaria: habilidades sociales. Trabajo de investigación tutelada no publicado: Universidad de Granada.

Pereda, (1987). Psicología Experimental. Madrid: Pirámide.

Redondo, S.; Pueyo, A. (2007). Psicología de la delincuencia. Papeles del Psicólogo, 28, 3 (2007)187-195.

Rodríguez, R. I.; Luca de Tena, C. (2001). Programa de disciplina en enseñanza secundaria obligatoria ¿Cómo puedo mejorar la gestión y el control de mi aula? Málaga: Aljibe.

Ross, R. R.; Ross, R. D. (1995). Thinking straight: the reasoning and rehabilitation program for delinquency $y$ prevention and offender rehabilitation. Otawa (Canadá): Air Training and Publications.

Ross, R.; Fabiano, E.; Garrido, V. (1990). El pensamiento prosocial. El modelo cognitivo para la prevención y tratamiento de la delincuencia. Delincuencia (monográfico).

Seijo, D.; Mesa, M. C.; Mohamed, N.; Mohamed, L. (2004). Programa EHSCO: entrenamiento en habilidades sociales con alumnos de educación primaria. Comunicación presentada al tercer congreso Internacional Virtual de Psicología de la Educación, celebrado en Internet del 9 al 29 de febrero.

Seijo, D.; Novo, M.; Fariña, F.; Arce, R.; Mesa, M. C. (2004). Prevención de comportamientos disruptivos en contextos escolares: programa de intervención basado en el entrenamiento de habilidades sociocognitivas. Programa EHSCO. Informe final de investigación. Universidad de Granada: Inédito.

Seijo, D; Novo, M.; Arce, R; Fariña, F; Mesa, M. C. (2005a). Prevención de comportamientos disruptivos en contextos escolares. Programa de intervención basado en el entrenamiento de habilidades sociocognitivas (programa ESHCO). Guía de actividades para Educación Primaria. Granada: GEU.

Seijo, D; Novo, M.; Arce, R; Fariña, F; Mesa, M. C. (2005b). Prevención de comportamientos disruptivos en contextos escolares. Programa de intervención basado en el entrenamiento de habilidades sociocognitivas (programa ESHCO). Guía de actividades para educación secundaria. Granada: GEU.

Timmerman, I.; Emmelkamp, P. (2005). An Integrated CognitiveBehavioural Approach to the Aetiology and Treatment of Violence. Clinical Psychology and Psychotherapy, 12 (2005) 167-176.

Trianes, M. V.; Fernández-Figeros, G. (2001). Aprender a ser personas y convivir. Madrid: Desclée.

Vallés, A.; Vallés, C. (1994). Programa de refuerzo en Habilidades Sociales I, II y III. Madrid: EOS

Vallés, A.; Vallés, C. (1996). Programa de refuerzo en Habilidades Sociales I, II y III. Madrid: EOS.

Vallés, A.; Vallés, C. (1997). Programa de solución de conflictos interpersonales I y II. Madrid: EOS. 
1 Program for improving socio-cognitive skills in elementary and secondary students from different cultural backgrounds in the Autonomous City of Melilla ${ }^{2}$ Doctora.

Universidad de Santiago de Compostela (España).

Email: mariadolores.seijo@usc.es

${ }^{3}$ Doctora.

Universidad de Santiago de Compostela (España).

Email: mercedes.novo@usc.es

${ }^{4}$ Doctora.

Universidad de Granada (España).

Email: lafu@ugr.es 